OPEN ACCESS

Edited by:

David Hansel,

Paris Descartes University, France

Reviewed by:

Germán Mato,

Centro Atomico Bariloche, Argentina

Takuma Tanaka,

Shiga University, Japan

${ }^{*}$ Correspondence:

Isao T. Tokuda

isao@fc.ritsumei.ac.jp

Received: 21 July 2016 Accepted: 30 September 2016

Published: 18 October 2016

Citation:

Kada H, Teramae J-N and Tokuda IT (2016) Effective Suppression of

Pathological Synchronization in

Cortical Networks by Highly

Heterogeneous Distribution of Inhibitory Connections.

Front. Comput. Neurosci. 10:109

doi: 10.3389/fncom.2016.00109

\section{Effective Suppression of Pathological Synchronization in Cortical Networks by Highly Heterogeneous Distribution of Inhibitory Connections}

\author{
Hisashi Kada ${ }^{1}$, Jun-Nosuke Teramae ${ }^{2}$ and Isao T. Tokuda ${ }^{1 *}$ \\ 1 Department of Mechanical Engineering, Ritsumeikan University, Kusatsu-Shi, Japan, ${ }^{2}$ Department of Bioinformatic \\ Engineering, Graduate School of Information Science and Technology, Osaka University, Suita, Japan
}

Even without external random input, cortical networks in vivo sustain asynchronous irregular firing with low firing rate. In addition to detailed balance between excitatory and inhibitory activities, recent theoretical studies have revealed that another feature commonly observed in cortical networks, i.e., long-tailed distribution of excitatory synapses implying coexistence of many weak and a few extremely strong excitatory synapses, plays an essential role in realizing the self-sustained activity in recurrent networks of biologically plausible spiking neurons. The previous studies, however, have not considered highly non-random features of the synaptic connectivity, namely, bidirectional connections between cortical neurons are more common than expected by chance and strengths of synapses are positively correlated between pre- and postsynaptic neurons. The positive correlation of synaptic connections may destabilize asynchronous activity of networks with the long-tailed synaptic distribution and induce pathological synchronized firing among neurons. It remains unclear how the cortical network avoids such pathological synchronization. Here, we demonstrate that introduction of the correlated connections indeed gives rise to synchronized firings in a cortical network model with the long-tailed distribution. By using a simplified feed-forward network model of spiking neurons, we clarify the underlying mechanism of the synchronization. We then show that the synchronization can be efficiently suppressed by highly heterogeneous distribution, typically a lognormal distribution, of inhibitory-to-excitatory connection strengths in a recurrent network model of cortical neurons.

Keywords: spontaneous firing, cortical network, lognormal distribution, excitatory and inhibitory connections, heterogeneity, synchronization

\section{INTRODUCTION}

Sustained asynchronous irregular activity of cortical neurons is commonly observed in cell cultures (Gross et al., 1982; Plenz and Aertsen, 1996; Marom and Shahaf, 2002), in vitro (Mao et al., 2001; Shu et al., 2003b), and in vivo (Timofeev et al., 2000) even in the absence of external stimuli. The spontaneous asynchronous activity is, as a ground state of the cortex, assumed to be involved in various significant computations realized in cortex, including sensory perception (Arieli et al., 1996; Tsodyks et al., 1999), working memory (Fuster, 1995; Wang, 2002; Compte, 2006), signal processing 
(Kenet et al., 2003), and transmissions (Destexhe and Contreras, 2006; Kumar et al., 2010). While the underlying mechanism for the cortical network to generate and maintain the spontaneous asynchronous state has not been fully understood yet, theoretical studies of random networks with balanced excitatory and inhibitory activities showed that random networks of leaky integrate-and-fire neurons without external inputs can realize the asynchronous irregular state under a certain condition (Vogels and Abbott, 2005; Kumar et al., 2008).

Considering the latest findings of heterogeneous features of synaptic strengths of cortical neurons (Song et al., 2005; Lefort et al., 2009; Avermann et al., 2012; Buzsáki and Mizuseki, 2014), recent numerical and theoretical studies revealed that the asynchronous irregular state is robustly and spontaneously realized in networks of biologically plausible leaky integrate-andfire neurons with largely relaxed condition from the previous ones (Teramae et al., 2012; Ikegaya et al., 2013; Kriener et al., 2014). Moreover, asynchronous activities of neurons realized in these studies faithfully share various properties with the sustained activity actually observed in vivo, such as high-irregularity (Softky and Koch, 1993; Stiefel et al., 2013), extremely low firing rate (Hromádka et al., 2008; Mizuseki and Buzsáki, 2013), highconductance membrane potential with large fluctuation (Wilson and Kawaguchi, 1996; Destexhe et al., 2001), and persistent UP state of membrane potential (Steriade et al., 2001; Destexhe et al., 2003; Shu et al., 2003a). In these models, amplitudes of excitatory postsynaptic potentials (EPSP) for excitatory-toexcitatory connections follow a long-tailed distribution, such as the lognormal distribution, with many weak and a few extremely strong synapses, which allows networks to generate and stably maintain the asynchronous irregular activity (Teramae et al., 2012).

Another significant feature of synaptic connectivity of local cortical circuit (Song et al., 2005), however, has been rarely considered in the previous studies of spontaneous asynchronous state. Namely, the synaptic connectivity in local cortical circuit is highly non-random. Bidirectional connections between cortical neurons are reported to exist more commonly than expected by chance in a random network and strengths of synapses are positively correlated between pre- and postsynaptic neurons, implying that strong synapses are more clustered than the majority of weak synapses. The clustered connectivity between strong synapses may excessively enhance mutual excitation among neurons in these clusters, which may force the spontaneous activity to increase its firing rate and as a consequence induce pathological synchronization among spikes. It remains unclear how the cortical network suppresses the pathologically strong synchronization induced by the clustered synaptic connectivity with the long-tailed EPSP distribution.

In this study, we propose a plausible mechanism for suppressing the pathological synchronization in cortical networks with clustered synaptic connectivity with the longtailed EPSP distribution. First, we reveal that the synchronization is indirectly induced by common synaptic inputs from inhibitory-to-excitatory connections rather than directly induced by the excessive mutual excitation among the clustered neurons. Next, we show that highly heterogeneous distribution of synaptic strengths for inhibitory-to-excitatory connections, which is similar to the heterogeneous connectivity experimentally observed for excitatory-to-excitatory connections (Song et al., 2005; Lefort et al., 2009; Avermann et al., 2012; Buzsáki and Mizuseki, 2014), efficiently suppresses the pathological synchronization. We also numerically confirm that a moderate amount of heterogeneity given by Gaussian distribution of the inhibitory synaptic strengths is not sufficient to suppress the synchronization. Moreover, we show that heterogeneity of the synaptic strengths in neither excitatory-to-inhibitory nor inhibitory-to-inhibitory connections effectively suppresses the synchronization. This result suggests a novel role of the heterogeneous inhibitory-to-excitatory connections with extremely strong inhibition (Miles and Wong, 1984; Chapeton et al., 2012) in realization of robust cortical state and its computation.

The rest of the paper is organized as follows. In Section 2 , we shortly describe the neuron and network models with explanation of analysis used in the paper. In Section 3.1, by introducing a finite correlation among EPSPs between bidirectionally connected excitatory neurons, we demonstrate that the experimentally observed synaptic connectivity, i.e., long-tailed EPSP distribution with the positive correlation, actually induce pathologic synchronization in the spontaneously sustained activity. In order to clarify the origin of the synchronization, in Section 3.2, we study a simplified feedforward network model, in which target neurons receive common synaptic inputs from neurons in the previous layer. Section 3.3 provides a possible mechanism to suppress pathological synchronization and recover stable asynchronous irregular state by introducing a highly heterogeneous distribution to inhibitory connections. Finally, in Section 4, we summarize our results and discuss possible relationship of our findings to cortical organization.

\section{METHODS}

\subsection{Single Neuron Model}

The dynamics of individual neuron is described by a conductance-based leaky integrate-and-fire model:

$$
\frac{d v}{d t}=-\frac{1}{\tau_{m}}\left(v-V_{L}\right)-g_{E}\left(v-V_{E}\right)-g_{I}\left(v-V_{I}\right),
$$

where $v$ represents membrane potential, $\tau_{m}$ is membrane time constant, and $V_{L}, V_{E}$, and $V_{I}$ are reversal potential of leak, excitatory and inhibitory postsynaptic currents, respectively. We use $\tau_{m}=20 \mathrm{~ms}$ for excitatory neurons, $\tau_{m}=10 \mathrm{~ms}$ for inhibitory neurons, $V_{L}=-70, V_{E}=0$, and $V_{I}=-80 \mathrm{mV}$. The excitatory and inhibitory synaptic conductances normalized by the membrane capacitance, $g_{E}$ and $g_{I}$, evolve with the following equation:

$$
\frac{d g_{X}}{d t}=-\frac{g_{X}}{\tau_{s}}+\sum_{j} G_{X, j} \sum_{s_{j}} \delta\left(t-s_{j}-d_{j}\right), \quad X=E, I,
$$

where the indices $X=E$ and $X=I$ are for excitatory and inhibitory conductances, respectively. $\delta(t)$ represents the delta 
function, $G_{X, j}, d_{j}$, and $s_{j}$ are synaptic weight, delay, and spike timing of synaptic input from the $j$-th neuron, respectively. The decay time constant $\tau_{s}$ is set to $2 \mathrm{~ms}$ for both excitatory and inhibitory conductances. The synaptic delays $d_{j}$ are chosen randomly from a uniform distribution between $d_{0}-1$ and $d_{0}+1$ $\mathrm{ms}$, where the mean value is set as $d_{0}=2 \mathrm{~ms}$ for excitatory-toexcitatory connections and $d_{0}=1 \mathrm{~ms}$ for the others. The spike threshold is $V_{t h r}=-50 \mathrm{mV}$ and $v$ is reset to $V_{r}=-70 \mathrm{mV}$ after the spiking. The refractory period is $1 \mathrm{~ms}$. These parameters are based on the ones of Teramae et al. (2012). The Euler's method is used to integrate the differential Equations $(1,2)$ with a time step of $0.01 \mathrm{~ms}$.

\subsection{Organization of Recurrent Network Model of Cortex}

The network model consists of $N_{E}=10,000$ excitatory neurons and $N_{I}=2000$ inhibitory neurons. For pairs of excitatory neurons, probabilities of unidirectional connections and bidirectional connections are $P_{u n i}=0.123$ and $P_{b i}=$ 0.0542, respectively, based on the physiological measurements (Song et al., 2005). Synaptic weights $G_{E, j}(j \in E)$ for unidirectional connections are distributed such that the amplitudes of EPSPs $x$ measured from the resting membrane potential obey a lognormal distribution,

$$
p(x)=\frac{\exp \left[-(\log x-\mu)^{2} / 2 \sigma_{N}^{2}\right]}{\sqrt{2 \pi} \sigma_{N} x},
$$

where $\mu$ and $\sigma_{N}$ represent mean and standard deviation of the variable's natural logarithm. We use values $\mu-\sigma_{N}^{2}=\log (0.2)$ and $\sigma_{N}^{2}=1.0$ to replicate experimentally observed long-tailed distributions of the amplitudes of EPSPs (Song et al., 2005). Any unrealistic value of $G_{E, j}$ that gives amplitude of EPSP larger than $20 \mathrm{mV}$ is discarded and we select a new value from the distribution.

In order to introduce the positive correlation of synaptic strengths measured between a pair of bidirectionally connected excitatory neurons, synaptic weights $G_{j, k}$ and $G_{k, j}(j, k \in E$ : bidirectionally connected excitatory neurons) are chosen such that amplitudes of the pair of EPSPs obey correlated random variables described as:

$$
\begin{aligned}
& x_{1}=\exp \left[\mu+\sigma_{N}\left(\sqrt{1-a} y_{1}+\sqrt{a} y_{3}\right)\right], \\
& x_{2}=\exp \left[\mu+\sigma_{N}\left(\sqrt{1-a} y_{2}+\sqrt{a} y_{3}\right)\right],
\end{aligned}
$$

where $y_{1}, y_{2}$, and $y_{3}$ are independent Gaussian variables with zero mean and unit variance. Each of $x_{1}$ and $x_{2}$ obeys the lognormal distribution of Equation (3), where their correlation is derived analytically as $R=\left\{e^{a \sigma_{N}^{2}}-1\right\} /\left\{e^{\sigma_{N}^{2}}-1\right\}$. By changing the parameter $a$, the correlation $R$ can be controlled. Physiologically measured value of the correlation is, for instance, $R=0.36$ for rat visual cortex (Song et al., 2005).

Based on the previous study (Teramae et al., 2012), constant values of $G_{E, j \in I}=0.018, G_{I, j \in E}=0.002$, and $G_{I, j \in I}=0.0025$ are used for excitatory-to-inhibitory, inhibitory-to-excitatory, and inhibitory-to-inhibitory connections, respectively. The connection probabilities of excitatory-to-inhibitory, inhibitoryto-excitatory and inhibitory-to-inhibitory neurons are $P_{E I}=$
$P_{b i}+P_{\text {uni }} / 2=0.1157, P_{I E}=P_{I I}=\left(N_{E} / N_{I}\right) P_{E I}=0.5785$, respectively. Excitatory-to-excitatory synaptic transmissions fail with a rate $p_{E}=b /(b+E P S P)$, where $b=0.1 \mathrm{mV}$ (Lefort et al., 2009). In order to initiate spontaneously maintained ongoing state, we apply transient external Poisson spike trains with 1 $\mathrm{Hz}$ to all neurons only during the initial duration of $100 \mathrm{~ms}$. The total simulation time including the initial transient is set to $2100 \mathrm{~ms}$. From time interval between 500 and $2100 \mathrm{~ms}$, dynamic quantities, e.g., firing frequencies, index of synchronized firing, strength of common inhibitory input as explained in the followings, are computed.

\subsection{Feed-Forward Network Model}

A feed-forward network model consisting of two layers of neurons is constructed to study the underlying mechanisms of correlation-induced synchrony. The input layer consists of 10, 000 excitatory and 2000 inhibitory neurons, whereas the output layer consists only of 1000 excitatory neurons. The connection probabilities from excitatory and inhibitory neurons in the input layer to neurons in the output layer are $P_{E E}=0.1157$ and $P_{I E}=0.5785$, respectively. The connection weights are set to be the same with those of the recurrent network model explained in the previous subsection.

Inhibitory neurons in the input layer generate mutually correlated Poisson spike trains as follows. First, we generate a single Poisson process with a constant firing rate and make each spike of the process be shared by randomly chosen $1 \%$ of the inhibitory neurons. The random selections are different from one another. Then, in addition to the randomly shared spikes, each neuron generates independent Poisson spikes so that its firing rate becomes $30 \mathrm{~Hz}$ in average. Frequency of the shared spikes ranges from 0 to $25 \mathrm{~Hz}$. Excitatory neurons in the input layer generate independent Poisson spike trains, where their firing rate is adjusted so that excitatory neurons in the output layer fire with an average rate of $3 \mathrm{~Hz}$.

\subsection{Index of Synchronized Firing}

We quantify the level of synchronization between spikes based on the cross-correlogram (CCG) of randomly chosen 1000 neurons. The CCG is calculated as a histogram of spike-time intervals (bin size: $1 \mathrm{~ms}$, time lag: $\pm 20 \mathrm{~ms}$ ) for all pairs of the 1000 neurons. Since existence of a peak in the CCG indicates synchronized firings, we define the synchronization index as a normalized height of the peak as

$$
S I=\frac{M-A}{M},
$$

where $M$ is the maximum and $A$ is the average of the CCG.

\subsection{Strength of Common Inhibitory Input}

As an index to measure the cause of synchrony, the strength of the common inhibitory input to excitatory neurons is quantified. The common inhibitory input is derived by time series of the inhibitory synaptic conductances $g_{I}$ averaged over excitatory neurons as $\left\{\bar{g}_{I}\left(t_{i}\right): i=1,2, \ldots, T\right\}$. Then, as the quantity to 
measure the signal strength, standard deviation of the averaged signal is computed with respect to the sampling time points as

$$
C I=\sqrt{\frac{1}{T} \sum_{i}^{T}\left\{\bar{g}_{I}\left(t_{i}\right)-\left\langle\bar{g}_{I}\right\rangle\right\}^{2}},
$$

where $\left\langle\bar{g}_{I}\right\rangle=\frac{1}{T} \sum_{i}^{T} \bar{g}_{I}\left(t_{i}\right)$ stands for time-average of the common inhibitory input.

\section{RESULTS}

\subsection{Correlation-Induced Pathological Synchronization in the Recurrent Network Model of Cortex}

We first evaluate synchronized firing of spontaneously sustained state in the recurrent network model of cortical circuits. We prepare networks with various values of correlation $R$ between EPSP amplitudes of bi-directionally connected pair of excitatory neurons. We then numerically solve Equations (1) and (2) on these networks to obtain the ongoing firing state and measure their synchronization indices (Figure 1A). In networks with low bidirectional correlation, $R<0.25$, the synchronization index is low, $S I \simeq 0.05$, where asynchronous ongoing activity is realized in a stable manner. Firing rate of the ongoing state is also kept low in this region (Figures 1E,F). The synchronization index, however, rapidly increases as the correlation $R$ increases from $R \simeq 0.25$. In the network with $R=0.35$, i.e., the value close to experimentally observed one for rat visual cortex, the synchronization index reaches $S I=0.19$, which is significantly higher than the one, $S I \simeq 0.05$, observed with low correlation. Figures 1C,D shows the raster plot of the ongoing state in the networks with $R=0.0$ and 0.35 . The spike timings are highly correlated among neurons in the network with $R=0.35$, whereas uncorrelated neural firings are observed with $R=0.0$. This implies that normal firing state of the cortex is replaced by pathological synchronization among neurons as bidirectional correlation is introduced to the model networks, even though the bidirectional connections are considered biologically plausible. It should be noted that the inhibitory neurons are more clearly synchronized with each other compared to the excitatory neurons even with $R=0.0$. This is however consistent with the experimental observation (Hasenstaub et al., 2005). Another note is that the large error-bar of Figure $\mathbf{1 A}$ at $R=0.25$ indicates coexistence of synchronization/desynchronization states around there.

In order to study details of the pathological synchronization, we measure temporal development of excitatory and inhibitory conductances on several excitatory neurons during the ongoing state in the networks with $R=0.0$ and 0.35 (Figure 2). We can see that inhibitory conductances are large and highly correlated with each other on different excitatory neurons especially when $R=0.35$ (Figure 2D), whereas excitatory conductances on the same neurons are weak and relatively distributed when $R=$ 0.0 (Figure 2C). This finding is also supported by Figure 1B which plots correlation between the inhibitory conductances as a function of $R$. For $R>0.25$, relatively high correlation is indicated among inhibitory conductances on different excitatory neurons. It has been reported in in vivo study (Hasenstaub et al., 2005) that inhibitory conductances tend to be synchronized with each other. The correlation, however, may not exceed 0.5 as shown in Figure 1B.

The present result therefore implies that strongly correlated inhibitory inputs, rather than excitatory inputs, drive neurons toward the pathological synchronization. It has been well studied that a common drive can easily induce strong synchronization among nonlinear systems including neurons (Mainen and Sejnowski, 1995; Teramae and Tanaka, 2004; Galán et al., 2006; de La Rocha et al., 2007; Doiron et al., 2016). In our network, the common inhibitory drive is kept weak as far as the correlation parameter $R$ is small, because firing rate of inhibitory neurons is kept low for a small $R$ (Figures 1E,F). As $R$ is increased, however, due to an increase in inhibitory firing rate as an outcome of excessive recurrent excitation mediated by the bidirectional excitatory connections, strength of the common inhibitory drive is increased and as a consequence induces strong pathological synchronization to the population of neurons in the network. The present finding agrees with other studies reporting that increase in firing rates leads to an increase in synchrony in cortical networks (Brunel and Hakim, 1999; Brunel, 2000; Mazzoni et al., 2008). Although, the network statistics are set to be closer to the real ones, the observed synchronized dynamics is significantly different from the asynchronous ongoing state observed in normal cortex.

\subsection{Suppression of Pathological Synchronization in a Simplified Feed-Forward Network Model}

Results of the previous subsection suggest a possibility that the pathological synchronization can be efficiently suppressed by reduction of strong correlation among inhibitory inputs on excitatory neurons. In order to systematically test the hypothesis and explore a possible and biologically plausible network structure that reduces the correlation among inhibitory inputs on excitatory neurons, here we consider a two-layer feedforward network. The input layer is composed of both inhibitory and excitatory neurons, whereas the output layer is composed only of excitatory neurons. In this network, we can artificially modulate correlated spike firings among the inhibitory input neurons.

We first confirm that the result of the previous subsection can be reproduced by increasing the correlated spike firings of inhibitory neurons in the input layer, which may induce synchronous firings of neurons in the output layer. As the frequency of the common inhibitory inputs is increased, the synchronization index of the output neurons increases almost linearly (Figure 3A). Indeed, the output spikes are less synchronized when the frequency of the common inhibitory input spikes is low (Figure 3B), while they show clear synchronization when the frequency is $25 \mathrm{~Hz}$ (Figure 3C).

Next, we explore a possible network mechanism that reduces the correlation of inhibitory inputs on output neurons to suppress their strong synchronization. Here, we focus on 
A

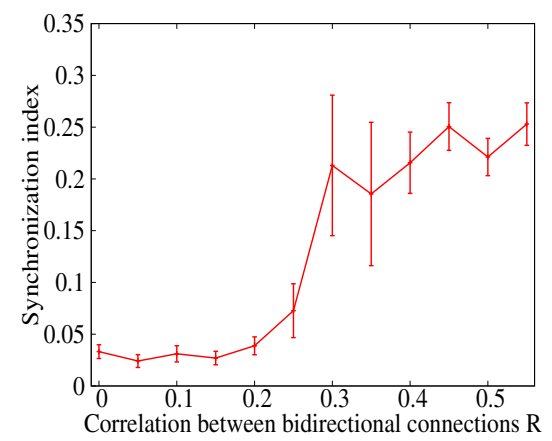

C

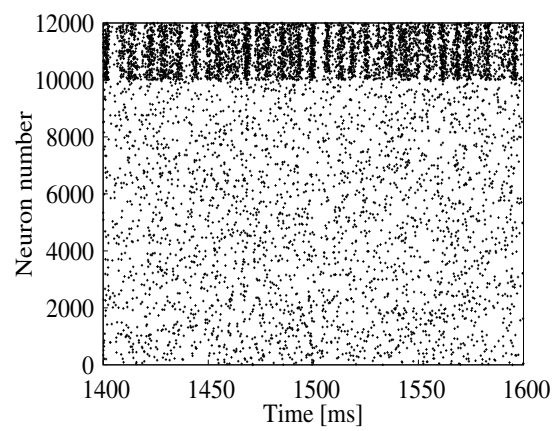

$\mathbf{E}$

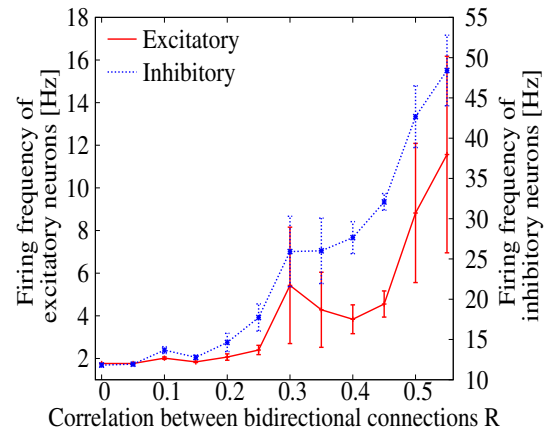

B

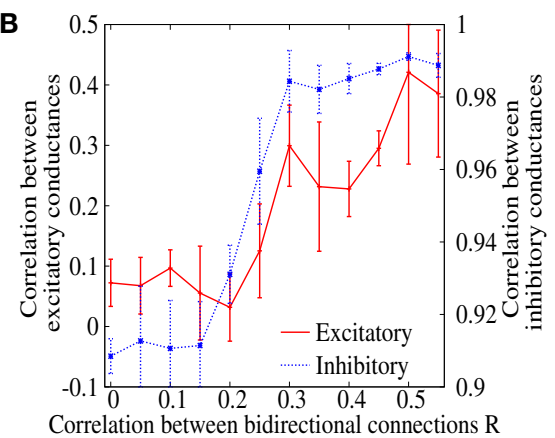

D

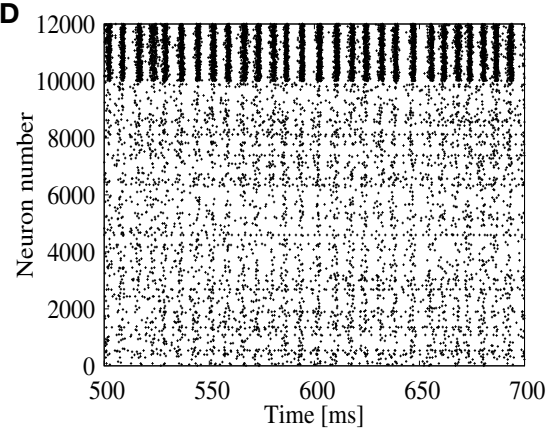

$\mathbf{F}$

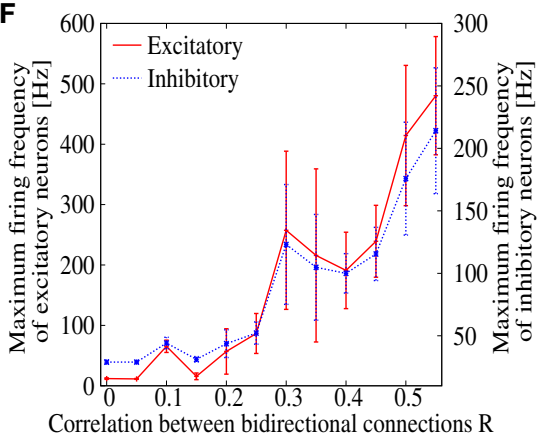

FIGURE 1 | (A) Dependence of the synchronization index on correlation parameter $R$, which measures correlation of EPSPs between bidirectionl connections. The error bars indicate standard deviation of 5 realizations started from different random initial conditions. (B) Dependence of the correlation between inhibitory (blue) and excitatory (red) synaptic conductances of excitatory neurons on the correlation parameter $R$. (C,D) Raster plot representing the firing pattern of $R=0$ (C) and $R=0.35$ (D). For excitatory neurons, spikes are indicated from neuron number 0 to 9999 , whereas they are indicated from neuron number 10,000 to 11,999 for inhibitory neurons. (E) Dependence of the mean firing frequencies of excitatory (red) and inhibitory (blue) neurons on the correlation parameter $R$. (F) Dependence of the maximum firing frequencies of excitatory (red) and inhibitory (blue) neurons on the correlation parameter $R$.

distribution of inhibitory connections on excitatory neurons. So far, we have assumed that strengths of inhibitory connections to output excitatory neurons are all the same, which may lead to a significant correlation among inhibitory inputs to them. Physiological experiments, however, reported that, in addition to heterogeneous EPSPs, inhibitory postsynaptic potentials (IPSPs) are also highly heterogeneous in the cortex (Miles and Wong, 1984; Holmgren et al., 2003; Chapeton et al., 2012). This heterogeneity can be a promising candidate to reduce the correlated inhibitory inputs on neurons and suppress the pathological synchronization in the cortex.

To study the above possibility, we measure the synchronization index among neurons in the output layer for three types of feed-forward networks with different amplitude distribution of the IPSPs, (1) constant (i.e., the same value is shared by all inhibitory connections), (2) the Gaussian distribution, and (3) the lognormal distribution. For the network of (1), we use $G_{I, j \in E}=0.002$, whereas, for the networks of (2) and (3), parameters of the synaptic distributions $G_{I, j \in E}$ are adjusted so that the mean IPSP amplitude keeps the same value as that of (1). We set frequency of the common inhibitory spike inputs to a high value, $25 \mathrm{~Hz}$, to mimic a possible correlated inhibitory spikes induced by the bidirectional correlation. Figure 4D shows the synchronization index and population firing rate of the neurons in the output layer. While the population firing rates are almost the same among the three networks, the synchronization index is low only for the network with the lognormal IPSP distribution. Indeed, the raster plots show that synchronized firing still remains in the networks with constant (Figure 4A) and 

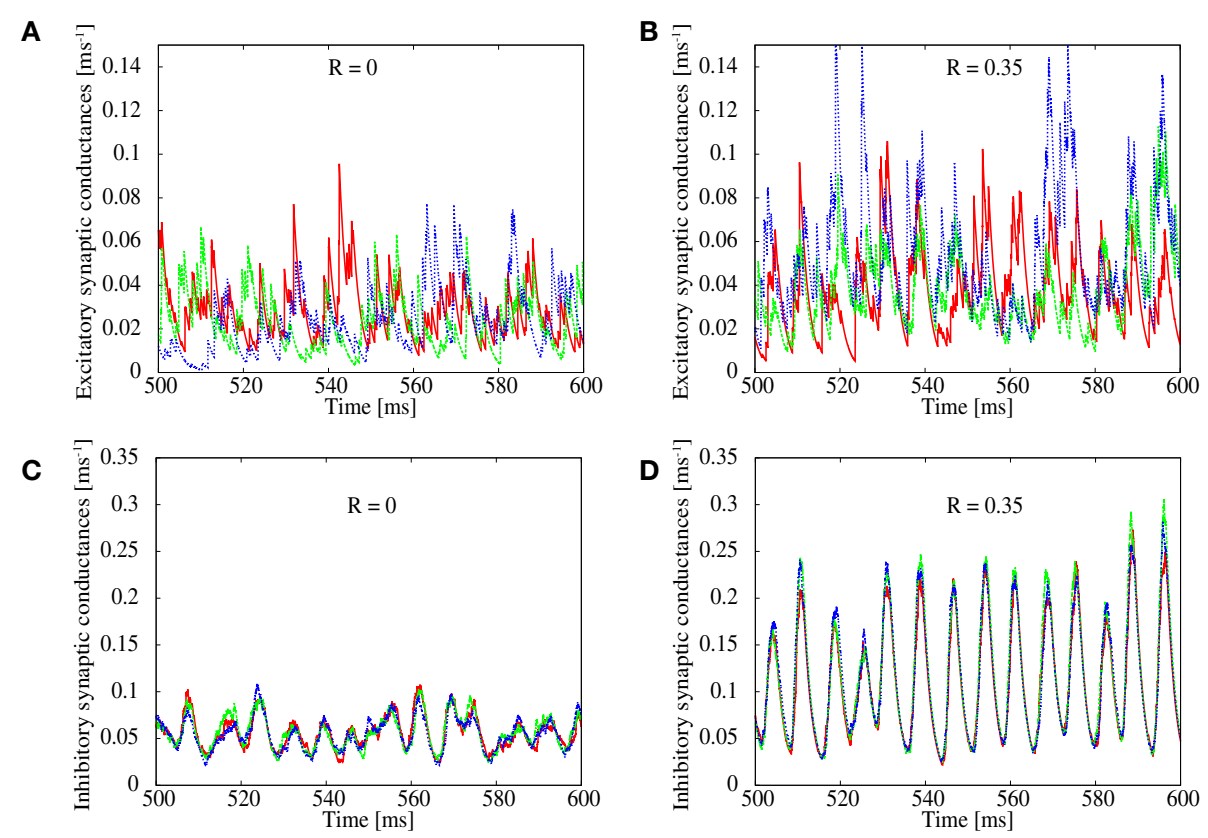

FIGURE 2 | Simultaneous plots of excitatory (top) and inhibitory (bottom) conductances of excitatory neurons. The correlation parameter is set to $R=0$ in (A,C) and $R=0.35$ in (B,D).

Gaussian (Figure 4B) IPSP distributions, whereas firing pattern of the lognormal IPSP network is asynchronous (Figure 4C).

In order to show distinctive advantage of the lognormal IPSP distribution over the Gaussian distribution to suppress the correlation-induced synchronization, now we measure synchronization index of the output neurons for ranges of parameters that control width of the distributions (Figures 5A,B). We vary the standard deviation, $\sigma_{G}$, of the Gaussian distribution and $\sigma_{N}$ of the lognormal distribution (see Equation 3) with keeping the mean IPSP amplitudes the same. As shown in Figures 5A,B, the synchronization index decreases only at the first range of $\sigma_{G}$ and stays at almost the same value even for sufficiently large values of $\sigma_{G}$ in the case of Gaussian distribution. On the contrary, the synchronization index continues to decrease in the full range of $\sigma_{N}$ for the lognormal distribution, and reaches a value $<0.1$, that is remarkably small, in the network with the lognormal IPSP distribution. These results imply that modest heterogeneity of IPSP amplitudes, i.e., the Gaussian distribution, is not sufficient to suppress the synchronization, while the highly heterogeneous IPSP distribution, such as the lognormal distribution, works well.

\subsection{Suppression of Pathological Synchronization in the Recurrent Network Model of Cortex}

In the previous subsection, we showed that the lognormal IPSP distribution efficiently suppresses the synchronization in the feed-forward network. Here, we apply the same strategy to the recurrent network, where correlation between bidirectional EPSPs results in pathological synchronization
$(R=0.35$; Figure 1A). In order to suppress the pathological synchronization, we introduce an additional lognormal distribution to the inhibitory-to-excitatory connections of the recurrent network (Figure 6A). In comparison, we also introduce a lognormal distribution to the excitatory-to-inhibitory connections (Figure 6B) or to the inhibitory-to-inhibitory connections (Figure 6C). Parameters of the lognormal distribution (i.e., $\mu, \sigma_{N}$ ) are adjusted so that the network maintains self-sustained activities and the neural firings are desynchronized. As shown in the raster plot, the synchronization is clearly suppressed in the case that the heterogeneity is introduced to the inhibitory-to-excitatory connections (Figure 6A). On the contrary, synchronization still remains in the case that the heterogeneity is introduced to the excitatoryto-inhibitory connections (Figure 6B). In the case that the heterogeneity is introduced to the inhibitory-to-inhibitory connections, the synchronization is also suppressed (Figure 6C). However, average firing rate of some neurons in the network is often extremely high, more than about $100 \mathrm{~Hz}$ (Figure 6D). In cortical networks, average firing rates of neurons in the resting state should be normally $<20 \mathrm{~Hz}$. Note that if we readjust parameters of the lognormal IPSP distributions to avoid such extremely high firing rate, it destabilizes the spontaneous firings and results in disappearance of the spontaneous firing state. Figures 6E,F summarize these results.

In order to see the robustness of suppressing the pathological synchronization by the additional lognormal distribution of the inhibitory-to-excitatory connection strengths, we measure the synchronization index of the spontaneous activity in recurrent networks with various correlation parameter 

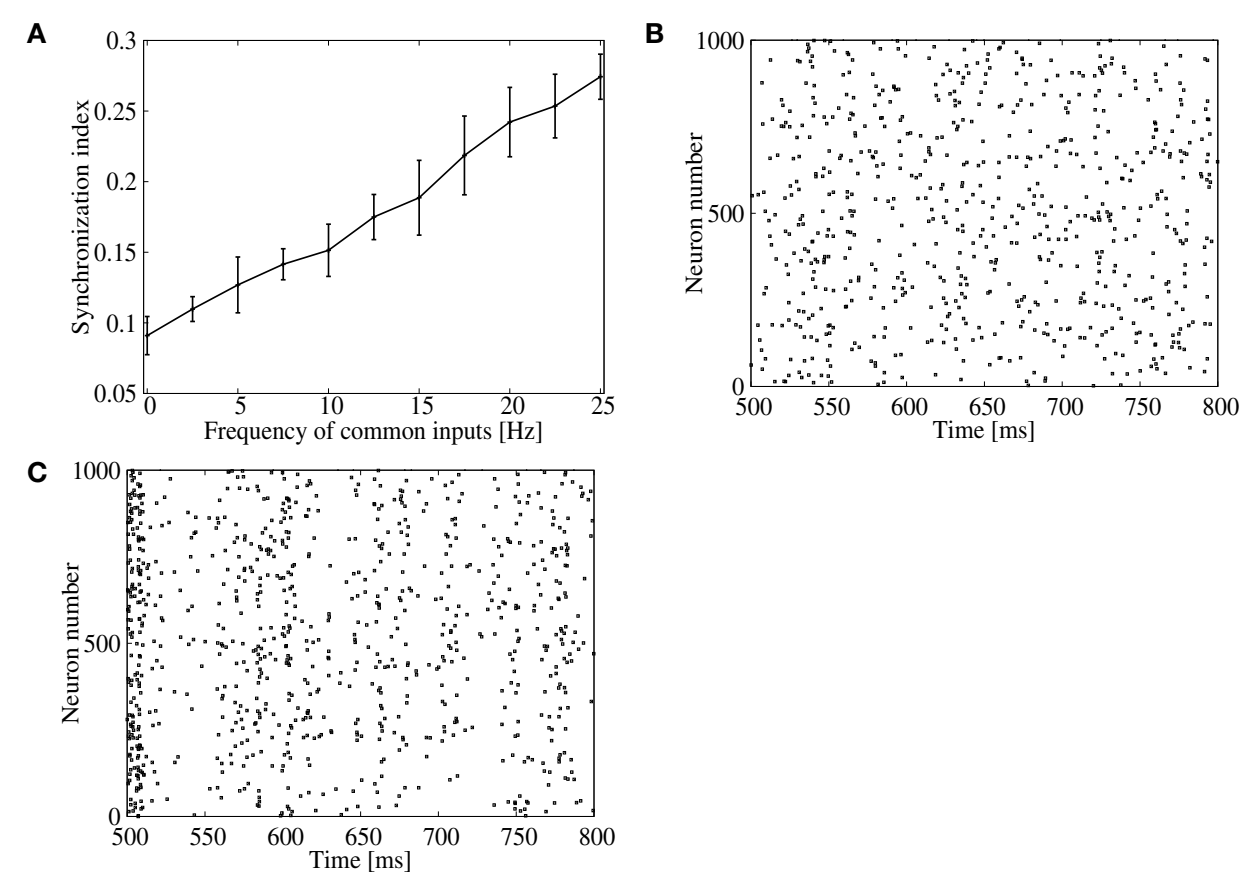

FIGURE 3 | Effect of inhibitory common inputs on synchronous firing of the feed-forward model. (A) Dependence of the synchronization index between excitatory neurons on frequency of common inputs. The error bars indicate standard deviation of 5 realizations started from different random initial conditions. (B,C) Raster plots for excitatory neurons in the output layer. No common inputs were injected in (B), whereas common inputs with a frequency of $25 \mathrm{~Hz}$ were injected in (C)

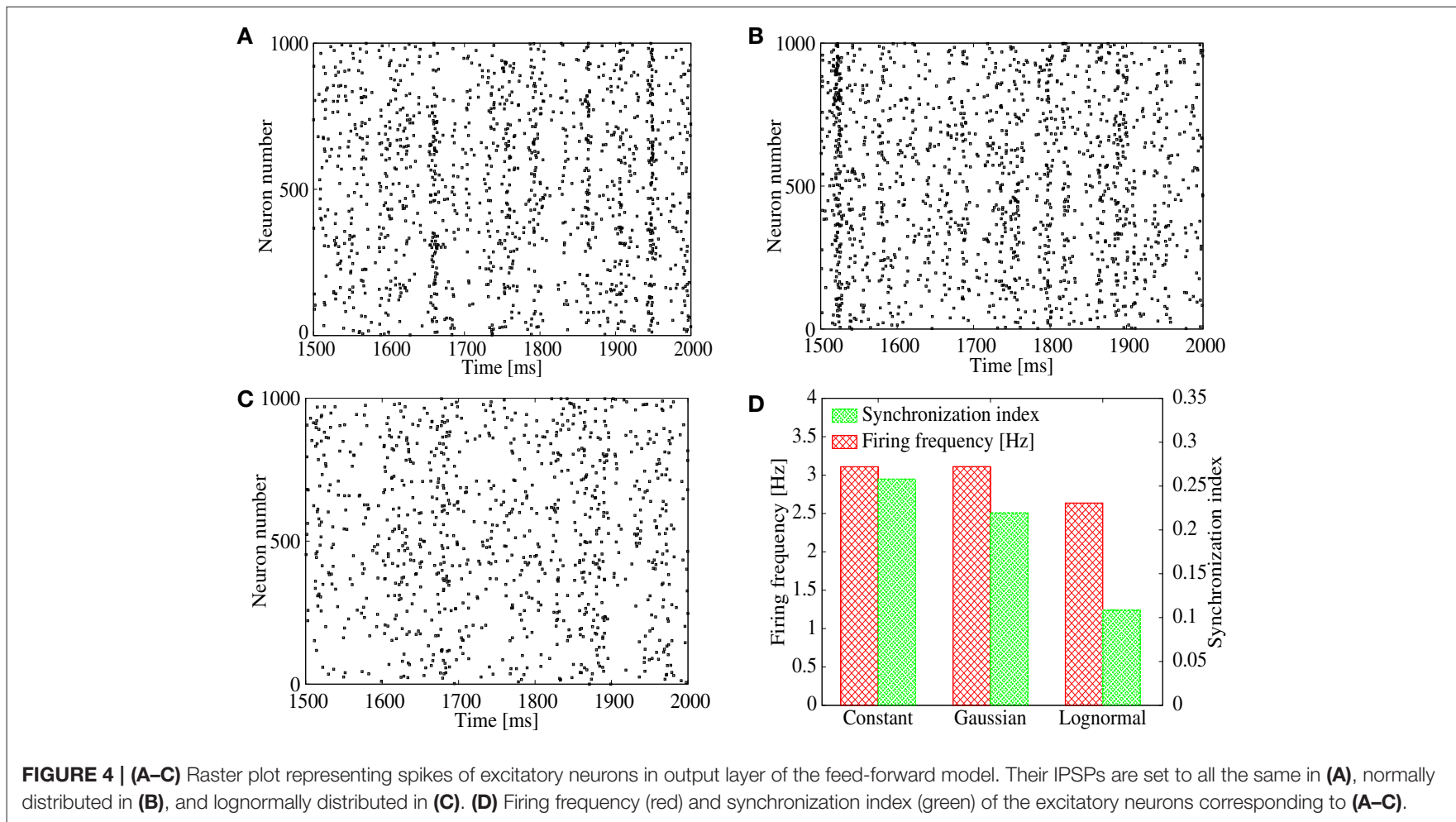

$R$. Figure 7A compares three cases: (i) in addition to the lognormal distribution in the excitatory-to-excitatory connections, no lognormal distribution is introduced (red), (ii) lognormal distribution is introduced to the inhibitory-toexcitatory connections (blue), and (iii) lognormal distribution is introduced to the excitatory-to-inhibitory connections 

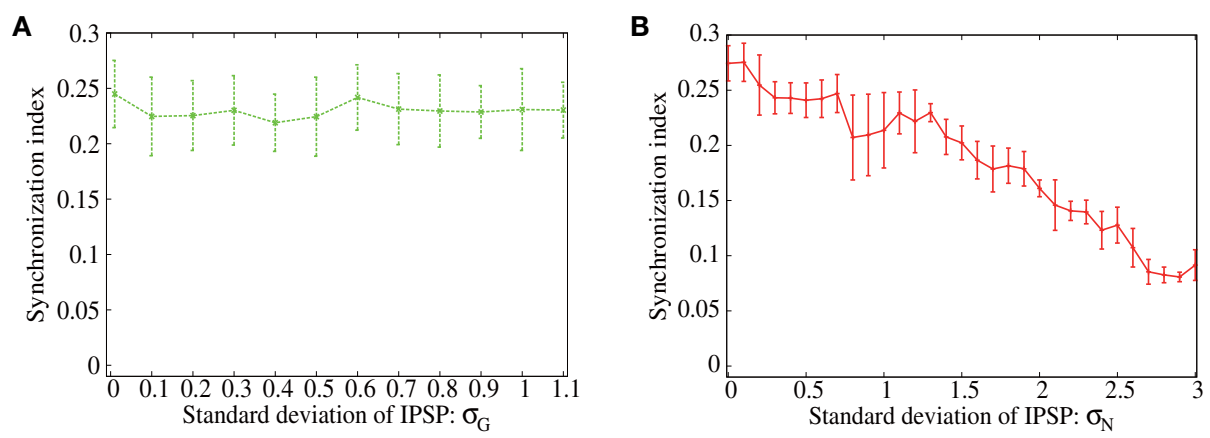

FIGURE 5 | (A,B) Dependence of the synchronization index between excitatory neurons on standard deviation of the IPSPs. The error bars indicate standard deviation of 5 realizations started from different random initial conditions. Frequency of the common inputs is set to $25 \mathrm{~Hz}$. The IPSPs are normally distributed in (A), while they are lognormally distributed in (B).

A

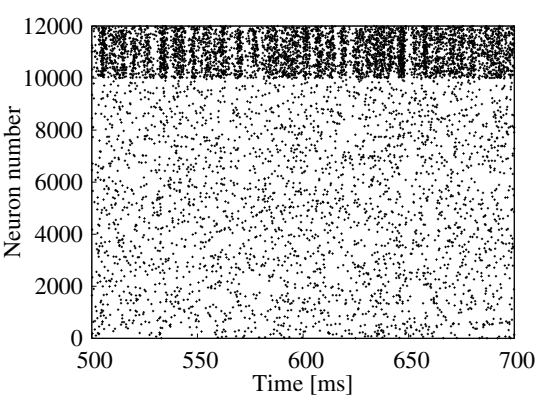

C

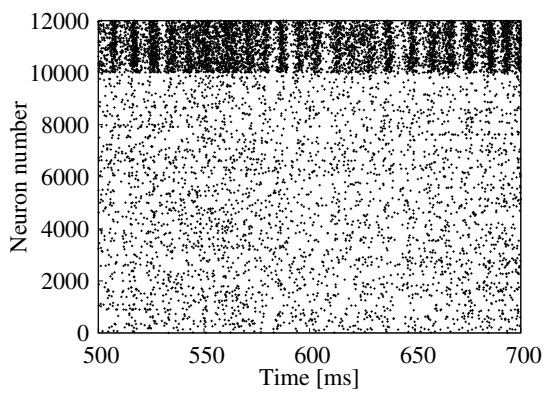

$\mathbf{E}$

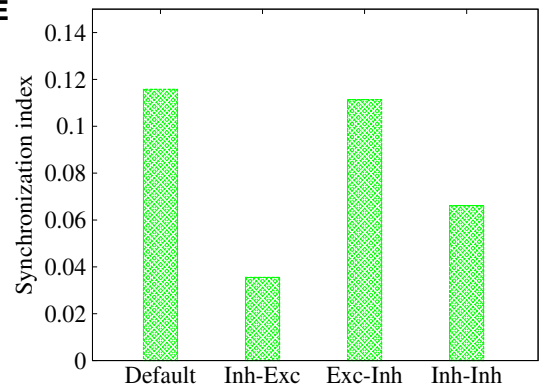

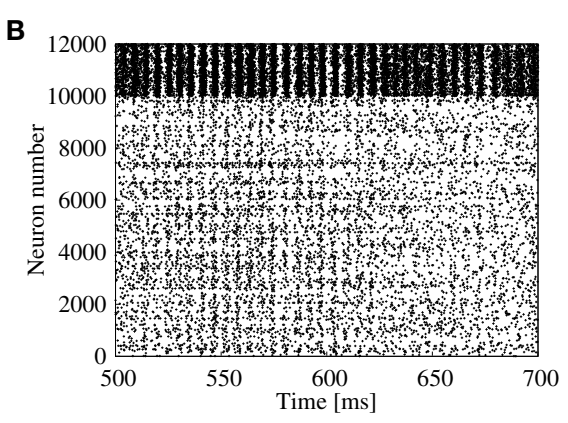

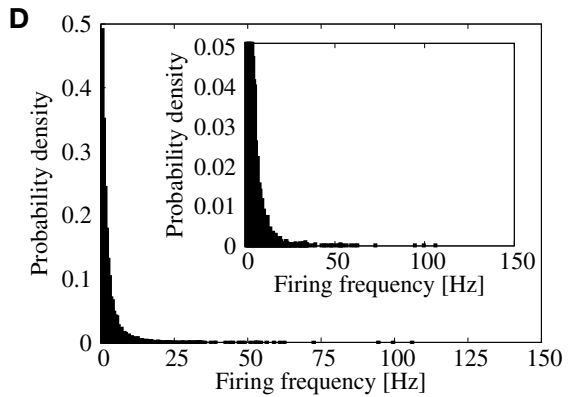

F

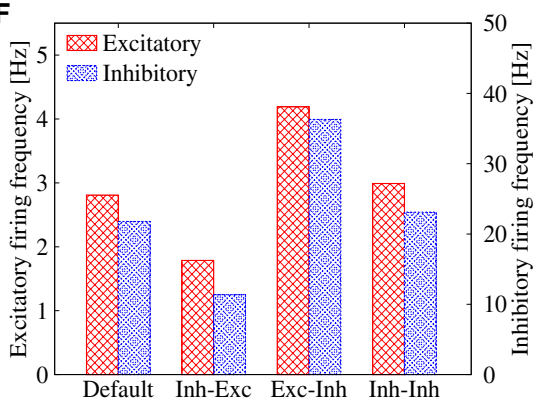

FIGURE 6 | (A-C) Raster plots representing the firing pattern of the cortical network model in case of $R=0.35$. For excitatory neurons, their spikes are indicated from neuron number 0 to 9999, whereas those of inhibitory neurons are indicated from neuron number 10, 000 to 11, 999 . In addition to the lognormally distributed excitatory-to-excitatory connections, lognormal distribution is introduced also to inhibitory-to-excitatory connections in (A), excitatory-to-inhibitory connections in (B), and inhibitory-to-inhibitory connections in (C). (D) Distribution of the firing frequencies of excitatory neurons corresponding to (C). The inset represents the enlarged graph. (E) Synchronization indices of the excitatory neurons corresponding to Figure 1A ("Default”), Panel (A) (“Inh-Exc"), Panel (B) (“Exc-Inh"), and Panel (C) ("Inh-Inh"). (F) Mean firing rates of the excitatory (red) and inhibitory (blue) neurons corresponding to Figure 1A ("Default”), Panel (A) ("Inh-Exc"), Panel (B) ("ExC-Inh"), and Panel (C) (“Inh-Inh"). 

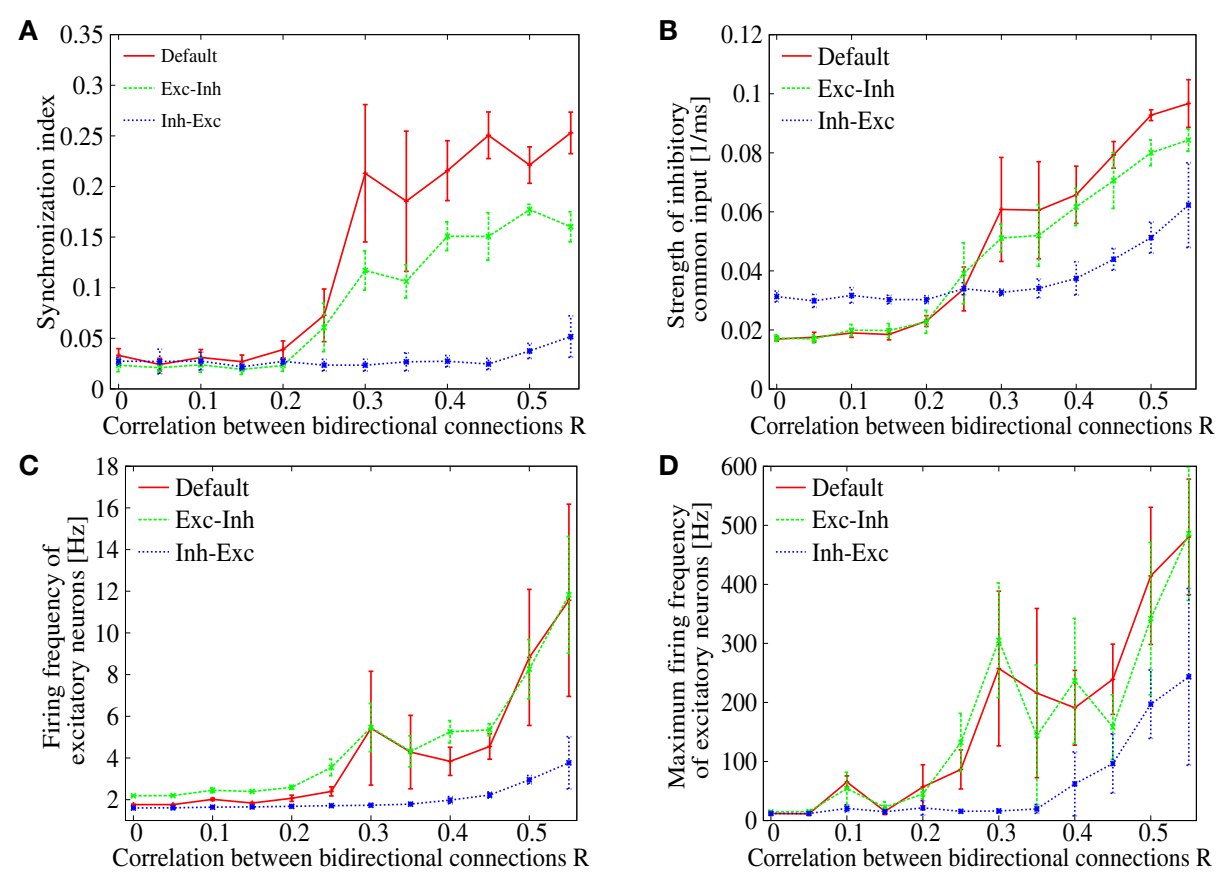

FIGURE 7 | (A) Dependence of the synchronization index on correlation parameter $R$. The red solid line is identical to Figure 1A ("Default"), whereas blue or green dotted line corresponds to the case that lognormal distribution is introduced to inhibitory-to-excitatory connections ("Inh-Exc") or excitatory-to-inhibitory ("Exc-Inh") connections. The error bars indicate standard deviation of 5 realizations started from different random initial conditions. (B) Dependence of the strength of the common inhibitory input $\mathrm{Cl}$ on the correlation parameter $R$. (C) Dependence of the mean firing frequencies of the excitatory neurons on the correlation parameter $R$. (D) Dependence of the maximum firing frequencies of the excitatory neurons on the correlation parameter $R$.

(green). In case (i), the synchronization index starts to increase rapidly as the correlation $R$ is increased from about 0.25 (as we have already seen in Figure 1A). Similarly, in case (iii), the synchronization index starts to increase around $R>0.25$. In contrast, in case (ii), the synchronization index is clearly suppressed over almost whole range of the correlation $R$. The lognormal distribution introduced to the inhibitory-to-excitatory connections, therefore, has a distinctive effect on desynchronization in the network model with biologically plausible features of non-random synaptic connectivity. Although, several studies reported that heterogeneities decrease synchrony in cortical networks (White et al., 1998; Golomb and Hansel, 2000; Neltner et al., 2000), no emphasis has been made on the importance of a long-tailed distribution, e.g., the lognormal distribution, as the form of heterogeneity.

The mechanism of desynchronization is considered to be due to the heterogeneity in the inhibitory-to-excitatory connections, which disperse the commonality of the spike inputs and weaken the strength of the common inhibitory drive. Figure 7B shows dependence of the common input strength $C I$ on the correlation parameter $R$. In cases (i) and (iii), the common input strength increases monotonously as the correlation $R$ is increased (red and green). The common input strength stayed in a low level up to $R<0.2$ and then it increases rapidly as the correlation $R$ is further increased. This coincides very well with the onset of synchronization, in which the level of synchrony increases in
Figure 7A (red and green). In contrast, only a minor increase in the common input strength is observed in case (ii) (blue line of Figure 7B). Thus, the strength of the common input drive $C I$ may provide a good index to measure the causality of the synchronized dynamics. As shown in Figures 7C,D, firing rates of the excitatory neurons are kept in a low level in case (ii) (blue). The firing rates, on the other hand, reach to an abnormal range in the other two cases (red and green). This implies that the heterogeneity in the inhibitory-to-excitatory connections is effective not only for suppressing the synchrony but also for maintaining the firing rates in a normal frequency range.

\section{DISCUSSION}

In this paper, pathological synchronization of spontaneous firings and a possible mechanism to suppress them in a cortical network model with biologically plausible nonrandom features of connectivity has been investigated. While the networks with coexistence of many weak and a few extremely strong excitatory synapses, or the lognormal EPSP distribution, can sustain spontaneous asynchronous irregular firing, introduction of experimentally observed positive correlation between bidirectional excitatory connections destabilizes the asynchronous activity and induces pathological synchronization among spontaneous neural firings. We show 
that the additional lognormal distribution in inhibitory-toexcitatory connections efficiently suppresses the pathological synchronization. The heterogeneous IPSPs induce a large response variability among excitatory neurons even when they are driven by highly correlated inhibitory inputs. In the studies of sensory neurons, effects of correlated input to neurons have been widely studied. For instance, it has been reported that the correlated inputs induce synchronous firings among neurons (Mainen and Sejnowski, 1995; Galán et al., 2006; de La Rocha et al., 2007; Doiron et al., 2016). Our present study reveals that correlated input from inhibitory neurons, that is indirectly strengthened by the bidirectional excitatory correlations, is the major cause of the pathological synchronization in networks with the long-tailed and correlated connectivity of excitatory synapses. We confirm validity of the additional heterogeneity in inhibitory connections in both a feed-forward and a biologically plausible model of the cortical network. Our result provides a functional role of highly heterogeneous distribution of strengths in inhibitory connections (Miles and Wong, 1984; Chapeton et al., 2012) that avoids pathological activity and ensures stable asynchronous irregular state in the cortex.

It should be noted that the present study is based on numerical simulations, the results of which may depend upon the parameter setting of the neural network model. The present setting, however, has been configured cautiously in good correspondence with mathematical analysis in the previous study (Teramae et al., 2012) and the newly added correlation between bidirectional connections are based on the physiological measurement (Song et al., 2005). Moreover, tendency of the network dynamics that the inhibitory neurons are more strongly synchronized with each other than the excitatory neurons (Figure 1C) is consistent with the experimental observation (Hasenstaub et al., 2005). It is therefore reasonable to consider that synchronized inputs from the inhibitory neurons are the primary cause of inducing synchronous firings among the excitatory neurons. It might be plausible to conclude that introduction of the heterogeneity to the inhibitory connections, which weakens the strength of the common inhibitory drive, provides an efficient way of suppressing the synchronous firings of the excitatory neurons.

It could be a significant future subject to study relationships between bidirectional excitatory correlation, heterogeneity among IPSPs, and synchronized firings observed during developmental process of the brain. It has been reported that cortical neurons show synchronized spontaneous activity both in vivo (Golshani et al., 2009) and in vitro (Corlew et al., 2004) in neonatal stage. Interestingly, the key differences in network structure between premature and mature brains are closely related to our results. First, connection probability between neurons in premature brain is generally higher than that in mature brain (Chechik et al., 1999; Paolicelli et al., 2011). The dense connectivity may imply highly correlated bidirectional couplings between excitatory neurons, where the connectivity gets sparse in developmental process probably due to synaptic plasticity. Second, GABAergic interneurons give excitatory actions to postsynaptic neurons rather than inhibitory actions in immature brain (Ben-Ari, 2002; Owens and Kriegstein, 2002). The excitatory actions may increase neural firing frequencies as observed in the pathological firings of our model. Reversal potential of GABA, which is initially in the level of depolarized membrane potential, shifts to mature hyperpolarized level in developmental process. Thus, the network structure such as correlated connections and heterogeneous inputs may largely change during development. How developmental process and synaptic plasticity balance between correlation of bidirectional connectivity and inhibitory heterogeneity to compatibly realize spontaneous "synchronous" firing of immature brain as well as spontaneous "asynchronous" irregular firing of mature brain is one of the most important future subjects.

Another subject that may relate to the present study is epileptic seizure. Decrease in GABAergic inhibition is known to cause epilepsy in experiment, for instance, in temporal lobe (Cossart et al., 2001; Wendling et al., 2002). Possible underlying mechanism of the epilepsy can be an imbalance between excitatory and inhibitory connections, which are well balanced in normal cortex (McCormick and Contreras, 2001). In addition to the collapse of the net balance, our results also suggest that decreased heterogeneity in inhibitory connections can be an alternative cause of the pathological synchronization. Even when the net strength of inhibitory inputs are balanced with that of excitatory ones, if the heterogeneity is broken by some reason, it can strengthen a potential correlation among the inhibitory inputs to neurons and bring the whole network to a strong synchronization with high firing rate. Such abnormal state should be quite similar to epileptic seizure. While the relationship between pathological synchronization of the model and actual epileptic seizure in cortex remains unclear, it must be worth while to study possible roles of heterogeneity of inhibitory connection strengths to prevent epilepsy in the brain.

\section{AUTHOR CONTRIBUTIONS}

JT and IT designed the study and discussed the results. HK performed the model simulations and analyzed the data. JT, IT, and $\mathrm{HK}$ wrote the text.

\section{FUNDING}

This work was partially supported by the Ministry of Internal Affairs and Communications with a contract entitled "R\&D for fundamental technology for energy-saving network control compatible to changing communication status" in FY2014, as well as by KAKENHI (No. 25430028, No. 26286086, No. 25293053, No. 16K00343, No. 16H01719). 


\section{REFERENCES}

Arieli, A., Sterkin, A., Grinvald, A., and Aertsen, A. (1996). Dynamics of ongoing activity: explanation of the large variability in evoked cortical responses. Science 273, 1868-1871.

Avermann, M., Tomm, C., Mateo, C., Gerstner, W., and Petersen, C. C. (2012). Microcircuits of excitatory and inhibitory neurons in layer $2 / 3$ of mouse barrel cortex. J. Neurophysiol. 107, 3116-3134. doi: 10.1152/jn.00917.2011

Ben-Ari, Y. (2002). Excitatory actions of gaba during development: the nature of the nurture. Nat. Rev. Neurosci. 3, 728-739. doi: 10.1038/nrn920

Brunel, N. (2000). Dynamics of sparsely connected networks of excitatory and inhibitory spiking neurons. J. Comput. Neurosci. 8, 183-208. doi: 10.1023/A:1008925309027

Brunel, N., and Hakim, V. (1999). Fast global oscillations in networks of integrateand-fire neurons with low firing rates. Neural Comput. 11, 1621-1671. doi: 10.1162/089976699300016179

Buzsáki, G., and Mizuseki, K. (2014). The log-dynamic brain: how skewed distributions affect network operations. Nat. Rev. Neurosci. 15, 264-278. doi: $10.1038 / \mathrm{nrn} 3687$

Chapeton, J., Fares, T., LaSota, D., and Stepanyants, A. (2012). Efficient associative memory storage in cortical circuits of inhibitory and excitatory neurons. Proc. Natl. Acad. Sci. U.S.A. 109, E3614-E3622. doi: 10.1073/pnas.1211467109

Chechik, G., Meilijson, I., and Ruppin, E. (1999). Neuronal regulation: a mechanism for synaptic pruning during brain maturation. Neural Comput. 11, 2061-2080. doi: 10.1162/089976699300016089

Compte, A. (2006). Computational and in vitro studies of persistent activity: edging towards cellular and synaptic mechanisms of working memory. Neuroscience 139, 135-151. doi: 10.1016/j.neuroscience.2005.06.011

Corlew, R., Bosma, M. M., and Moody, W. J. (2004). Spontaneous, synchronous electrical activity in neonatal mouse cortical neurones. J. Physiol. 560, 377-390. doi: 10.1113/jphysiol.2004.071621

Cossart, R., Dinocourt, C., Hirsch, J. C., Merchan-Perez, A., De Felipe, J., Ben-Ari, Y., et al. (2001). Dendritic but not somatic gabaergic inhibition is decreased in experimental epilepsy. Nat. Neurosci. 4, 52-62. doi: 10.1038/ 82900

de La Rocha, J., Doiron, B., Shea-Brown, E., Josić, K., and Reyes, A. (2007). Correlation between neural spike trains increases with firing rate. Nature 448, 802-806. doi: 10.1038/nature06028

Destexhe, A., and Contreras, D. (2006). Neuronal computations with stochastic network states. Science 314, 85-90. doi: 10.1126/science.1127241

Destexhe, A., Rudolph, M., Fellous, J.-M., and Sejnowski, T. J. (2001). Fluctuating synaptic conductances recreate in vivo-like activity in neocortical neurons. Neuroscience 107, 13-24. doi: 10.1016/S0306-4522(01)00344-X

Destexhe, A., Rudolph, M., and Paré, D. (2003). The high-conductance state of neocortical neurons in vivo. Nat. Rev. Neurosci. 4, 739-751. doi: $10.1038 / \mathrm{nrn} 1198$

Doiron, B., Litwin-Kumar, A., Rosenbaum, R., Ocker, G. K., and Josić, K. (2016). The mechanics of state-dependent neural correlations. Nat. Neurosci. 19, 383-393. doi: 10.1038/nn.4242

Fuster, J. M. (1995). Memory in the Cerebral Cortex. Cambridge, MA: MIT Press.

Galán, R. F., Fourcaud-Trocmé, N., Ermentrout, G. B., and Urban, N. N. (2006). Correlation-induced synchronization of oscillations in olfactory bulb neurons. J. Neurosci. 26, 3646-3655. doi: 10.1523/JNEUROSCI.4605-05.2006

Golomb, D., and Hansel, D. (2000). The number of synaptic inputs and the synchrony of large, sparse neuronal networks. Neural Comput. 12, 1095-1139. doi: 10.1162/089976600300015529

Golshani, P., Gonçalves, J. T., Khoshkhoo, S., Mostany, R., Smirnakis, S., and Portera-Cailliau, C. (2009). Internally mediated developmental desynchronization of neocortical network activity. J. Neurosci. 29, 1089010899. doi: 10.1523/JNEUROSCI.2012-09.2009

Gross, G. W., Williams, A. N., and Lucas, J. H. (1982). Recording of spontaneous activity with photoetched microelectrode surfaces from mouse spinal neurons in culture. J. Neurosci. Methods 5, 13-22. doi: 10.1016/0165-0270(82) 90046-2

Hasenstaub, A., Shu, Y., Haider, B., Kraushaar, U., Duque, A., and McCormick, D. A. (2005). Inhibitory postsynaptic potentials carry synchronized frequency information in active cortical networks. Neuron 47, 423-435. doi: 10.1016/j.neuron.2005.06.016
Holmgren, C., Harkany, T., Svennenfors, B., and Zilberter, Y. (2003). Pyramidal cell communication within local networks in layer $2 / 3$ of rat neocortex. J. Physiol. 551, 139-153. doi: 10.1113/jphysiol.2003.044784L

Hromádka, T., DeWeese, M. R., and Zador, A. M. (2008). Sparse representation of sounds in the unanesthetized auditory cortex. PLoS Biol. 6:e16. doi: 10.1371/journal.pbio.0060016

Ikegaya, Y., Sasaki, T., Ishikawa, D., Honma, N., Tao, K., Takahashi, N., et al. (2013). Interpyramid spike transmission stabilizes the sparseness of recurrent network activity. Cereb. Cortex 23, 293-304. doi: 10.1093/cercor/bhs006

Kenet, T., Bibitchkov, D., Tsodyks, M., Grinvald, A., and Arieli, A. (2003). Spontaneously emerging cortical representations of visual attributes. Nature 425, 954-956. doi: 10.1038/nature02078

Kriener, B., Enger, H., Tetzlaff, T., Plesser, H. E., Gewaltig, M.-O., and Einevoll, G. T. (2014). Dynamics of self-sustained asynchronous-irregular activity in random networks of spiking neurons with strong synapses. Front. Comput. Neurosci. 8:136. doi: 10.3389/fncom.2014.00136

Kumar, A., Rotter, S., and Aertsen, A. (2010). Spiking activity propagation in neuronal networks: reconciling different perspectives on neural coding. Nat. Rev. Neurosci. 11, 615-627. doi: 10.1038/nrn2886

Kumar, A., Schrader, S., Aertsen, A., and Rotter, S. (2008). The highconductance state of cortical networks. Neural Comput. 20, 1-43. doi: 10.1162/neco.2008.20.1.1

Lefort, S., Tomm, C., Sarria, J.-C. F., and Petersen, C. C. H. (2009). The excitatory neuronal network of the $\mathrm{c} 2$ barrel column in mouse primary somatosensory cortex. Neuron 61, 301-316. doi: 10.1016/j.neuron.2008.12.020

Mainen, Z. F., and Sejnowski, T. J. (1995). Reliability of spike timing in neocortical neurons. Science 268, 1503-1506. doi: 10.1126/science.7770778

Mao, B.-Q., Hamzei-Sichani, F., Aronov, D., Froemke, R. C., and Yuste, R. (2001). Dynamics of spontaneous activity in neocortical slices. Neuron 32, 883-898. doi: 10.1016/S0896-6273(01)00518-9

Marom, S., and Shahaf, G. (2002). Development, learning and memory in large random networks of cortical neurons: lessons beyond anatomy. Q. Rev. Biophys. 35, 63-87. doi: 10.1017/S0033583501003742

Mazzoni, A., Panzeri, S., Logothetis, N. K., and Brunel, N. (2008). Encoding of naturalistic stimuli by local field potential spectra in networks of excitatory and inhibitory neurons. PLoS Comput. Biol. 4:e1000239. doi: 10.1371/journal.pcbi.1000239

McCormick, D. A., and Contreras, D. (2001). On the cellular and network bases of epileptic seizures. Annu. Rev. Physiol. 63, 815-846. doi: 10.1146/annurev.physiol.63.1.815

Miles, R., and Wong, R. (1984). Unitary inhibitory synaptic potentials in the guinea-pig hippocampus in vitro. J. Physiol. 356, 97-113.

Mizuseki, K., and Buzsáki, G. (2013). Preconfigured, skewed distribution of firing rates in the hippocampus and entorhinal cortex. Cell Rep. 4, 1010-1021. doi: 10.1016/j.celrep.2013.07.039

Neltner, L., Hansel, D., Mato, G., and Meunier, C. (2000). Synchrony in heterogeneous networks of spiking neurons. Neural Comput. 12, 1607-1641. doi: 10.1162/089976600300015286

Owens, D. F., and Kriegstein, A. R. (2002). Is there more to gaba than synaptic inhibition? Nat. Rev. Neurosci. 3, 715-727. doi: 10.1038/nrn919

Paolicelli, R. C., Bolasco, G., Pagani, F., Maggi, L., Scianni, M., Panzanelli, P., et al. (2011). Synaptic pruning by microglia is necessary for normal brain development. Science 333, 1456-1458. doi: 10.1126/science.1202529

Plenz, D., and Aertsen, A. (1996). Neural dynamics in cortex-striatum coculturesii. spatiotemporal characteristics of neuronal activity. Neuroscience 70, 893-924. doi: 10.1016/0306-4522(95)00405-X

Shu, Y., Hasenstaub, A., Badoual, M., Bal, T., and McCormick, D. A. (2003a). Barrages of synaptic activity control the gain and sensitivity of cortical neurons. J. Neurosci. 23, 10388-10401.

Shu, Y., Hasenstaub, A., and McCormick, D. A. (2003b). Turning on and off recurrent balanced cortical activity. Nature 423, 288-293. doi: 10.1038 /nature01616

Softky, W. R., and Koch, C. (1993). The highly irregular firing of cortical cells is inconsistent with temporal integration of random epsps. J. Neurosci. 13, 334-350.

Song, S., Sjöström, P. J., Reigl, M., Nelson, S., and Chklovskii, D. B. (2005). Highly nonrandom features of synaptic connectivity in local cortical circuits. PLoS Biol. 3:e68. doi: 10.1371/journal.pbio.0030068 
Steriade, M., Timofeev, I., and Grenier, F. (2001). Natural waking and sleep states: a view from inside neocortical neurons. J. Neurophysiol. 85, 1969-1985.

Stiefel, K. M., Englitz, B., and Sejnowski, T. J. (2013). Origin of intrinsic irregular firing in cortical interneurons. Proc. Natl. Acad. Sci. U.S.A. 110, 7886-7891. doi: $10.1073 /$ pnas. 1305219110

Teramae, J.-N., and Tanaka, D. (2004). Robustness of the noise-induced phase synchronization in a general class of limit cycle oscillators. Phys. Rev. Lett. 93:204103. doi: 10.1103/PhysRevLett.93.204103

Teramae, J.-N., Tsubo, Y., and Fukai, T. (2012). Optimal spike-based communication in excitable networks with strong-sparse and weak-dense links. Sci. Rep. 2:485. doi: 10.1038/srep00485

Timofeev, I., Grenier, F., Bazhenov, M., Sejnowski, T. J., and Steriade, M. (2000). Origin of slow cortical oscillations in deafferented cortical slabs. Cereb. Cortex 10, 1185-1199. doi: 10.1093/cercor/10.12.1185

Tsodyks, M., Kenet, T., Grinvald, A., and Arieli, A. (1999). Linking spontaneous activity of single cortical neurons and the underlying functional architecture. Science 286, 1943-1946. doi: 10.1126/science.286.54 46.1943

Vogels, T. P., and Abbott, L. F. (2005). Signal propagation and logic gating in networks of integrate-and-fire neurons. J. Neurosci. 25, 10786-10795. doi: 10.1523/JNEUROSCI.3508-05.2005
Wang, X.-J. (2002). Probabilistic decision making by slow reverberation in cortical circuits. Neuron 36, 955-968. doi: 10.1016/S0896-6273(02)01092-9

Wendling, F., Bartolomei, F., Bellanger, J. J., and Chauvel, P. (2002). Epileptic fast activity can be explained by a model of impaired gabaergic dendritic inhibition. Eur. J. Neurosci. 15, 1499-1508. doi: 10.1046/j.1460-9568.2002.01985.x

White, J. A., Chow, C. C., Rit, J., Soto-Treviño, C., and Kopell, N. (1998). Synchronization and oscillatory dynamics in heterogeneous, mutually inhibited neurons. J. Comput. Neurosci. 5, 5-16. doi: 10.1023/A:1008841325921

Wilson, C. J., and Kawaguchi, Y. (1996). The origins of two-state spontaneous membrane potential fluctuations of neostriatal spiny neurons. J. Neurosci. 16, $2397-2410$.

Conflict of Interest Statement: The authors declare that the research was conducted in the absence of any commercial or financial relationships that could be construed as a potential conflict of interest.

Copyright (c) $2016 \mathrm{Kada}$, Teramae and Tokuda. This is an open-access article distributed under the terms of the Creative Commons Attribution License (CC BY). The use, distribution or reproduction in other forums is permitted, provided the original author(s) or licensor are credited and that the original publication in this journal is cited, in accordance with accepted academic practice. No use, distribution or reproduction is permitted which does not comply with these terms. 